\title{
OBSERVATIONS OF SPACE DEBRIS IN THE VICINITY OF ORBITS OF GLOBAL NAVIGATION SATELLITE SYSTEMS
}

\author{
I.V. Korobtsev \\ Institute of Solar-Terrestrial Physics SB RAS, \\ Irkutsk, Russia, korobtsev@iszf.irk.ru \\ T.G. Tsukker \\ Institute of Solar-Terrestrial Physics SB RAS, \\ Irkutsk, Russia,tania@iszf.irk.ru \\ M.N. Mishina \\ Institute of Solar-Terrestrial Physics SB RAS, \\ Irkutsk,Russia,mmish@iszf.irk.ru
}

\author{
V.E. Goryashin \\ Institute of Solar-Terrestrial Physics SB RAS, \\ Irkutsk,Russia,vgor@iszf.irk.ru \\ M.V. Eselevich \\ Institute of Solar-Terrestrial Physics SB RAS, \\ Irkutsk,Russia,mesel@iszf.irk.ru
}

\begin{abstract}
The problem of the amount and characteristics of space debris in the vicinity of orbits of Global Navigation Satellite Systems (GNSS) is of significant interest from the viewpoint of safe operation of these systems. Attempts have repeatedly been made to search for space debris fragments in a given region of orbits, but have not led to cataloging such objects. Only in 2018, eight space objects were discovered which were not related to active or inactive spacecraft or their launch elements. Photometrical and trajectory observations with optical telescopes are practically the only source of information about characteristics of such objects. The paper presents a summary of the design features and technical characteristics of the new AZT-
\end{abstract}

33VM telescope. We describe a technique for determining orbital parameters of non-cataloged space debris from optical measurements. We report the results of photometric observations of a space object, detected in the vicinity of orbits of the Global Navigation Satellite System GLONASS.

Keywords: space debris, Global Navigation Satellite System, wide-field telescope.

\section{INTRODUCTION}

In recent years, global navigation satellite systems (GNSS) produced in different countries for military and civilian purposes have been actively developed. The normal functioning of these systems becomes extremely important for a wide range of government institutions and private users worldwide. The basic functional element of any GNSS is a constellation of spacecraft (SC) orbiting Earth in specified orbits. The GNSS orbit region is a strategic space zone that requires accurate estimate of current space situation for regular and safe operation of navigation SC.

Currently, $\sim 80 \%$ of more than 20 thousands of objects monitored on near-Earth orbits are fragments of various spacecraft (space debris, SD) and decommissioned SC, which pose a serious threat to expensive operational satellites [ESA's Annual Space Environment Report, 2019]. Most of cataloged SD objects are in low Earth orbits, controlled by radars. Artificial high-apogee space objects (SO) are monitored using optical telescopes. A number of papers have addresses the problem of man-made space debris in the geostationary orbit [Schildknecht et al., 2001; Seitzer et al., 2004; Schildknecht, 2007; Kelecy et al., 2013; Eselevich et al., 2017], and only a few publications have dealt with noncataloged SD in medium-Earth orbits [Silha et al., 2017], in particular in the vicinity of GNSS orbits [Dick et al., 2009; Schildknecht et al., 2012]. Data on parameters of orbits of all active and defunct SC, as well as means for their removal in the vicinity of GNSS orbits are updated regularly in open SO catalogs. At the same time, this region of space is insufficiently explored in terms of the amount and characteristics of small-sized SD. Modern medium-diameter (100-200 cm) optical telescopes are capable of detecting and cataloging objects up to the 20th-21st magnitude, which in mediumEarth orbits corresponds to an $\sim 5 \mathrm{~cm}$ object with a reflection coefficient of 0.15 [McCue et al., 1971].

In 2016, the 1.6-meter wide-angle telescope AZT$33 \mathrm{VM}$ with a high limiting magnitude was put into operation. It is designed for surveillance of near-Earth space. This paper describes a technique for observing and determining orbital parameters of non-cataloged space objects detected during optical observations. We report on observations of small-sized SD found in the vicinity of GNSS orbits.

\section{WIDE-ANGLE TELESCOPE AZT-33VM}

Optical telescopes play a significant role in monitoring objects in near-Earth orbits. A wide field of optical image is necessary for near-real-time monitoring and detection of active SO and SD in the greatest possible area of space. Requirements for the limiting magnitude 
of wide-angle telescopes are quite high. For example, addressing the problems of monitoring near-Earth space requires a limiting magnitude up to 22 . Ackermann et al. [2014] provides six representative examples that encompass a number of currently available optical designs of wide-angle telescopes. Noteworthy among new dedicated survey telescopes are the following projects: SST, Pan-STARRS, ATLAS, MCAT, AZT 33VM.

In 2016 at the ISTP SB RAS Sayan Solar Observatory, the new wide-angle telescope AZT-33VM was put into operation. Its distinguishing feature is a large field of view at a moderate aperture, which allows us to survey and measure SOs with brightness up to the 20th21 st magnitude in a large area of space. One of the observation tasks of the telescope is to investigate the man-made space debris in near-Earth space. When designing the telescope, the positive experience in manufacturing the $1.6 \mathrm{~m}$ high-aperture primary mirror and the equatorial fork mount for the AZT-33IK telescope has been used [Kamus et al., 2002]. The telescope has the quasi-Ritchey-Chrétien design with a prefocal lens field corrector; the diameter of the primary mirror is $1.6 \mathrm{~m}$, the focal length is $5.6 \mathrm{~m}$, the angular field of view is $2.8^{\circ}$. The main monochromatic aberrations to be corrected in the given field of view are astigmatism and curvature. The optical power of the corrector was taken to be close to zero in order to minimize both the chromatisms. To reduce the diameter of the corrector's lenses in the AZT-33VM telescope [Denisenko et al., 2009], the inside focus position has been invoked (Figure 1 , right). In addition, this solution greatly decreases the central vignetting parameter ( 0.4 in diameter).

Large gradients of mirror asphericity impose stringent requirements on their relative position accuracy. Therefore, the design of the telescope involves active control of the position of the secondary mirror by means of hexapod. To improve the quality of the images obtained and to increase the sensitivity of the telescope to weak SOs, a stray light protection system not provided by the manufacturer has been developed and installed [Chuprakov et al., 2018].
Currently, the photodetector of the telescope is not fully implemented, therefore its field of view is $30 \times 30$ arcmin with a $2048 \times 2048$ CCD camera. After equipped with a mosaic photodetector, its working field of view will be $2.7^{\circ} \times 1.3^{\circ}$.

Characteristics of the positional accuracy of AZT$33 \mathrm{VM}$ observations in right ascension are 0.7 arcsec; in declination, 0.6 arcsec. Figure 2 exemplifies residual deviations in position of object No.139900 after polynomial approximation of measurements.

\section{METHOD OF EVALUATING ORBITAL PARAMETERS$$
\text { OF NON-CATALOGED SO }
$$$$
\text { DETECTED FROM OPTICAL }
$$$$
\text { OBSERVATIONS WITH AZT-33VM }
$$

Observations of near-Earth space pose a problem of determining the orbit of a newly discovered SO. As a rule, at the initial stage of measurements there is insufficient data to determine accurate orbital parameters, and during follow-up observations the object's orbit is defined more accurately. The measurements should be organized so that to determine the orbit of an object as quickly and accurately as possible, without losing the object. To address this problem, a suitable method has been developed.

The problem of determining the SO orbit from related measurements (in the absence of other information) is solved in three stages involving determination and improvement of a preliminary orbit, construction and improvement of an averaged orbit, and construction of an osculating orbit. To define the orbit more accurately, different versions of the method of differential correction of orbital elements are used which take into account various orbital perturbations. The method is based on minimizing the functional for differences between calculated and observed apparent angular coordinates of right ascension and declination [Abalakin et al., 1976].
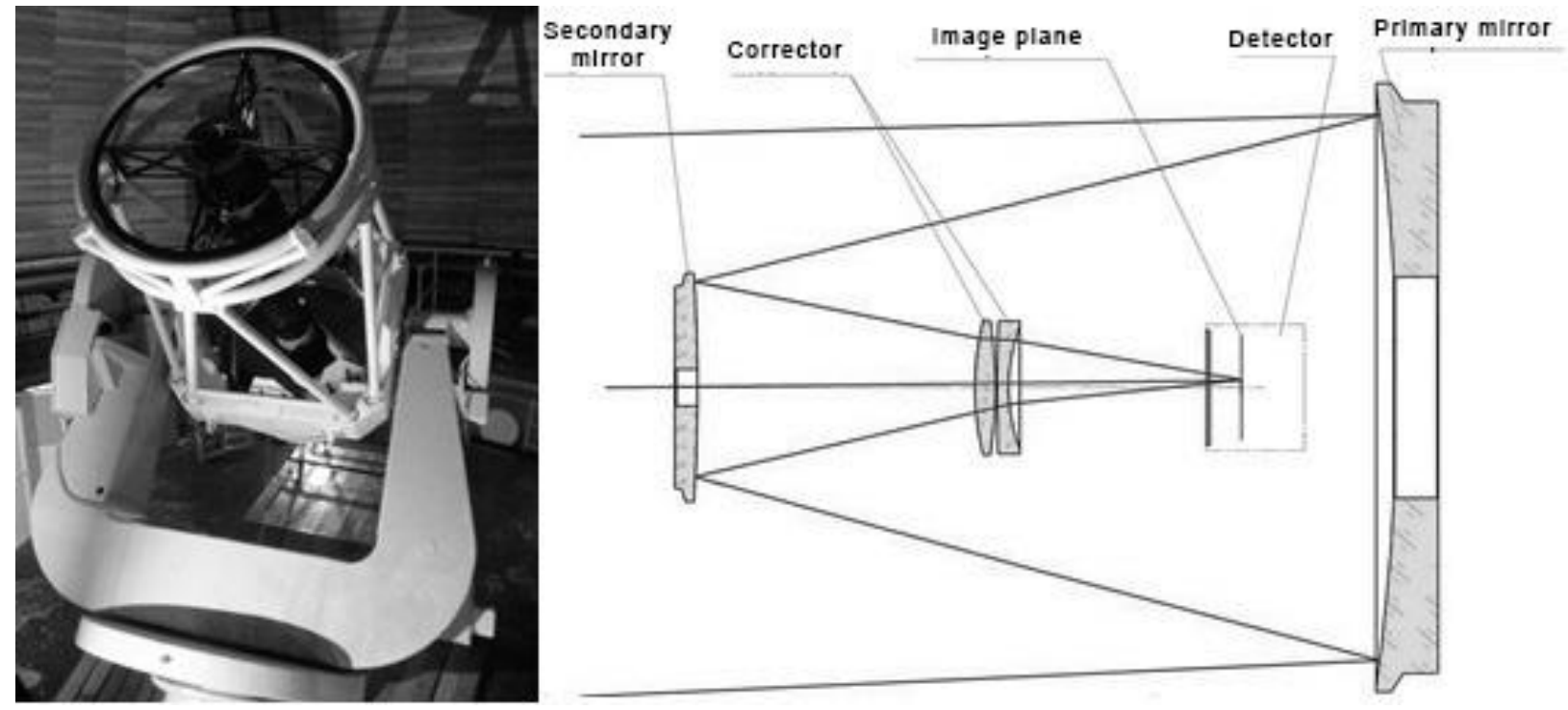

Figure 1. Telescope AZT-33VM inside the dome (left) and its optical layout 
As an initial approximation we take the circular unperturbed orbit obtained from two measurements maximally separated in time. Then, the initial orbit is sequentially improved using the other series of measurements. To predict the movement of the object during the next night, we calculate the averaged orbit from all observations of the first night, taking into account zonal harmonics of the geopotential (the main factor is the Earth compression). As an implementation of the model of SO movement, we use the open code SGP4 [Hoots et al., 1988]. Estimated accuracy of the prediction of SO position in the initial and averaged orbits depending on the interval of measurements is shown in Figure 3 by the example of object No. 3076.

The accuracy of the prediction of the SO position measured at a short arc (less than $10 \mathrm{~min}$ ) is seen to be rather low. Due to significant errors in elements of the initial orbit, the object can subsequently be detected only at a short time interval (15-20 min). With the measurement interval over $10 \mathrm{~min}$, the error in the estimated SO position decreases by almost two orders of magnitude and does not exceed 100 arcsec in the hour and a half interval of prediction. Detecting SO the next day requires a measurement interval of at least $60 \mathrm{~min}$. In this case, errors in the estimated SO position do not exceed the field of the telescope.

If the observation interval covers three or more days during which 4-5 series of measurements are made, we can calculate elements of the osculating orbit. The trajectory is calculated through numerical integration by the 16th-order Everhart method [Everhart, 1974; Bordovitsyna et al., 2016]. In this case, we take into account

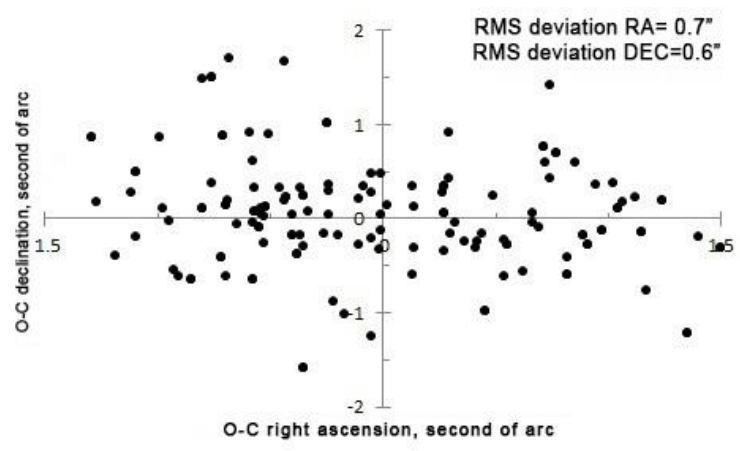

Figure 2. RMS deviations of positions of SO No. 139900

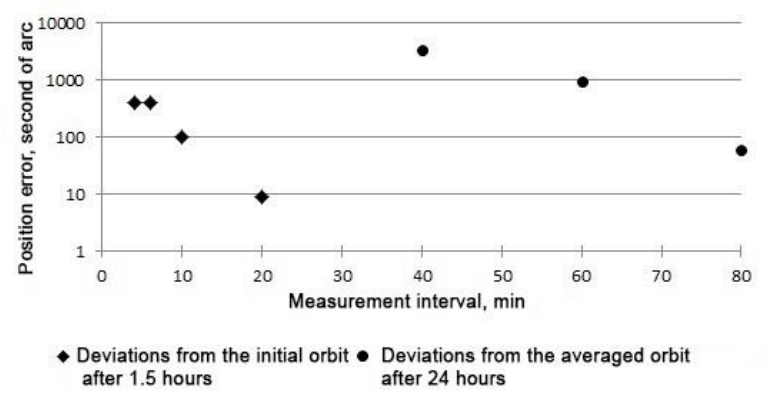

Figure 3. An error in estimated position of SO No. 3076 as a function of measurement interval. Diamonds denote deviations from the initial orbit in $1.5 \mathrm{hr}$ after the start of measurements; circles mark deviations from the averaged orbit the next day the following effects:

- perturbations associated with Earth's nonsphericity (to the 6th harmonic);

- lunar-solar perturbations;

- perturbations caused by light pressure.

Figure 4 exemplifies residuals between measurements and estimated positions of SO No.1509 along the osculating orbit calculated over the interval of three days. Standard deviations in right ascension are 0.3 arcsec; in declination, 0.6 arcsec.

The resulting osculating orbit allows us to predict the motion of an object from several days to a month. It becomes possible to solve the problem of tracking an object, and the object may be included in the SO catalog.

We can also identify the parameter of the area-tomass ratio $(A M R)$. Note that at $A M R<0.1$, its reliable calculation requires more than three days of observation.

\section{OVERVIEW OF THE NUMBER OF SD OBJECTS IN THE VICINITY OF GNSS AND PROBLEMS ASSOCI- ATED WITH THEIR DETECTION}

Until the beginning of 2018, there was no information about orbital parameters of small-sized SD fragments in the vicinity of GNSS orbits in open SO catalogs. Various researchers have attempted to undertake special surveys in order to identify non-cataloged SD and evaluate its amount in the vicinity of orbits of this type. Single SD objects were found, but no continuously tracked objects were cataloged. At the same time, there was data on 650 SD objects in the vicinity of geosynchronous orbits. At present, the published material including information about orbital parameters of highorbiting SD objects [Newsletters of PJSC "IAC Vympel", 2019] contains data on eight fragments in the vicinity of GNSS orbits. Six objects are in the vicinity of orbits of the Russian system GLONASS; and two, in the vicinity of the European system GALILEO. In total, 354 SOs have been cataloged in the vicinity of GNSS orbits: 256 are active and inactive SC, 90 are rocket bodies and various launch elements, 8 are SD objects. Figure 5 illustrates the distribution of all the objects over planes of orbits.

The primary factors limiting the large-scale detection and cataloging of SD in the vicinity of GNSS are apparent brightness and geometry of SO motion relative to groundbased optical instruments. Apparent brightness of SD objects in high orbits is an important parameter that directly determines the size of the telescope aperture necessary for recording solar emission reflected from a satellite. This parameter strongly depends on the size, orientation, and reflection coefficient of SO. SO apparent brightness can be represented by the relation [McCue et al., 1971]

$$
m_{\mathrm{SO}}=-26.58-2.5 \log \left(\frac{\operatorname{SaF}(\varphi)}{d^{2}}\right),
$$

where -26.58 is the apparent magnitude of the Sun, $S$ is the area of the reflecting surface of a satellite, $a$ is the reflection coefficient of SO surface, $F(\varphi)$ is the phase function, $d$ is the topocentric distance to the object. The reflection coefficient for the coating material in use can vary from 


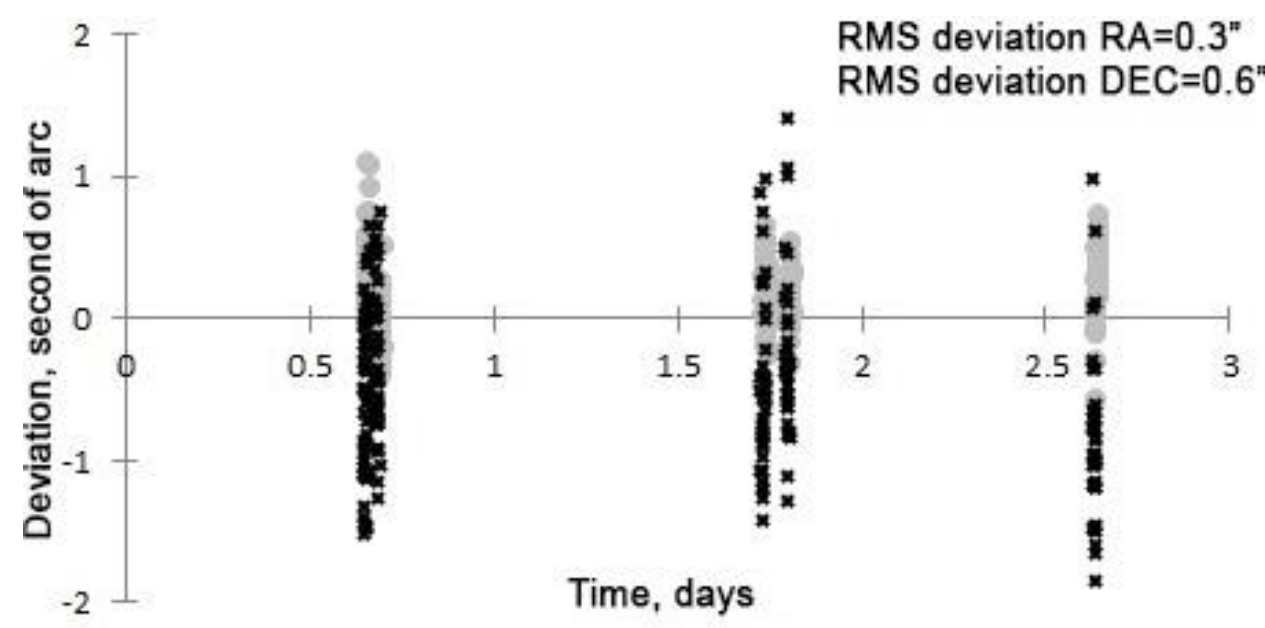

right ascension

$\times$ declination

Figure 4. Residuals between measurements and calculated angular coordinates along the osculating orbit over an interval of three days by the example of SO No.1509

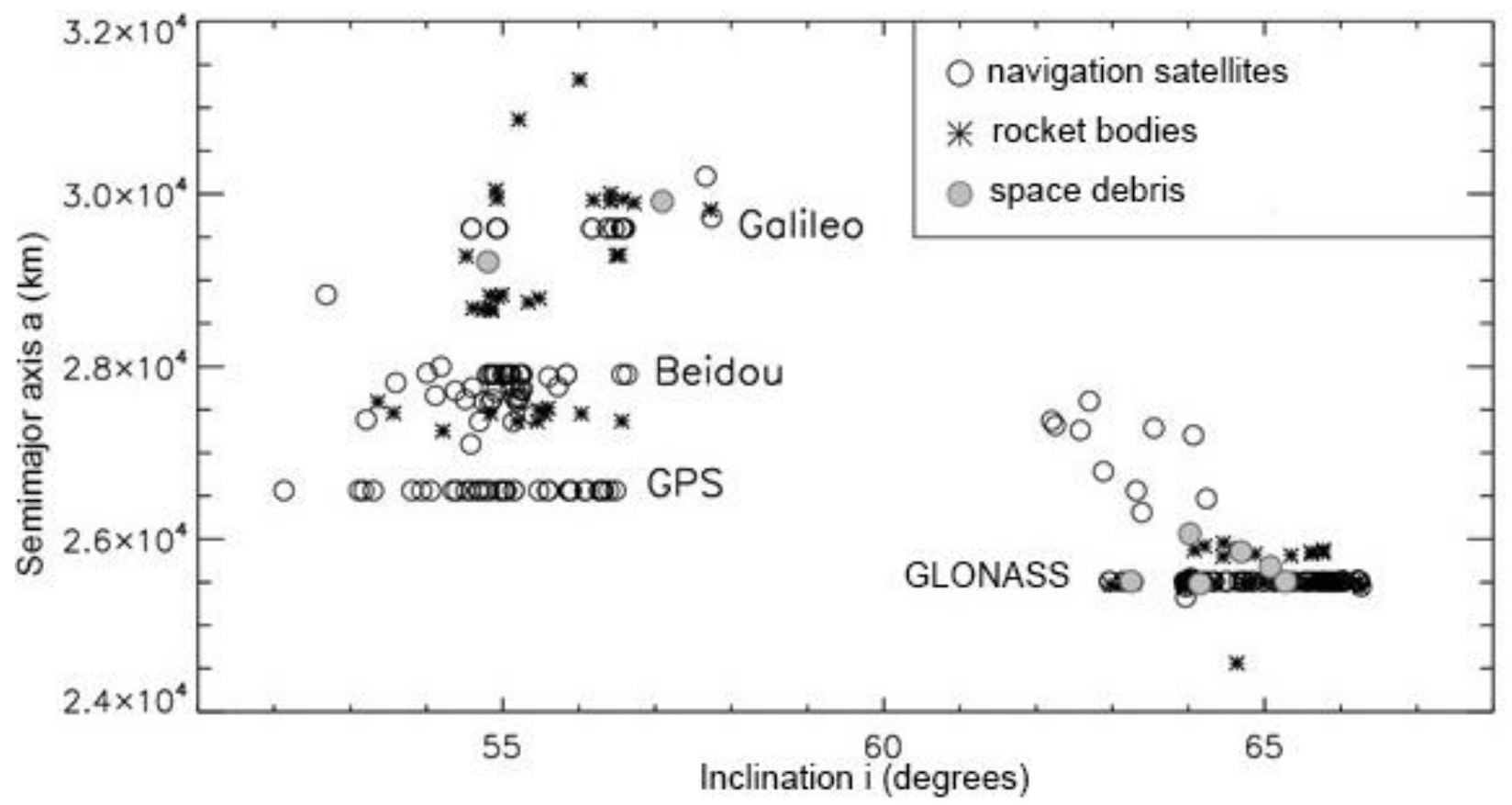

Figure 5. Distribution of cataloged SO in the vicinity of active GNSS

0.1 to 1 . A value from 0.1 to 0.18 is generally taken in estimations [Mulrooney et al., 2008]. For reflection from a diffuse sphere, the phase function $F(\varphi)$ is described by

$$
F(\varphi)=\frac{2}{3 \pi^{2}}[(\pi-\varphi) \cos \varphi+\sin \varphi],
$$

where $\varphi$ is the angle in the Sun - space object - observer system.

Thus, a $10 \mathrm{~cm}$ object near GNSS orbits at a zero phase angle and a reflection coefficient of 0.15 will have a brightness of the $\sim 18$ th magnitude.

As a quantitative criterion of precision measurement of light fluxes from astronomical sources, we take the level of the signal-to-noise ratio, defined by the formula [Howell, 2006]:

$$
\frac{S}{N}=\frac{N_{m}}{\sqrt{N_{m}+n_{\text {pix }}\left(N_{\mathrm{S}}+N_{\mathrm{D}}+N_{\mathrm{R}}^{2}\right)}},
$$

where $N_{m}$ is the number of electrons from an object of magnitude $m, N_{\mathrm{S}}$ is the number of electrons from the sky background per one pixel, $N_{\mathrm{D}}$ is the number of electrons from the detector's dark current per pixel, $N_{\mathrm{R}}^{2}$ is the number of electrons per pixel from the detector readout noise, $n_{\text {pix }}$ is the number of pixels of the detector, occupied by the object image. Figure 6 exemplifies a calculated signal-tonoise ratio for SO of the 18th magnitude when they move at different speeds in the field of AZT-33VM. The sky background for the calculation is taken to be 20 magnitude/arcsec ${ }^{2}$. 


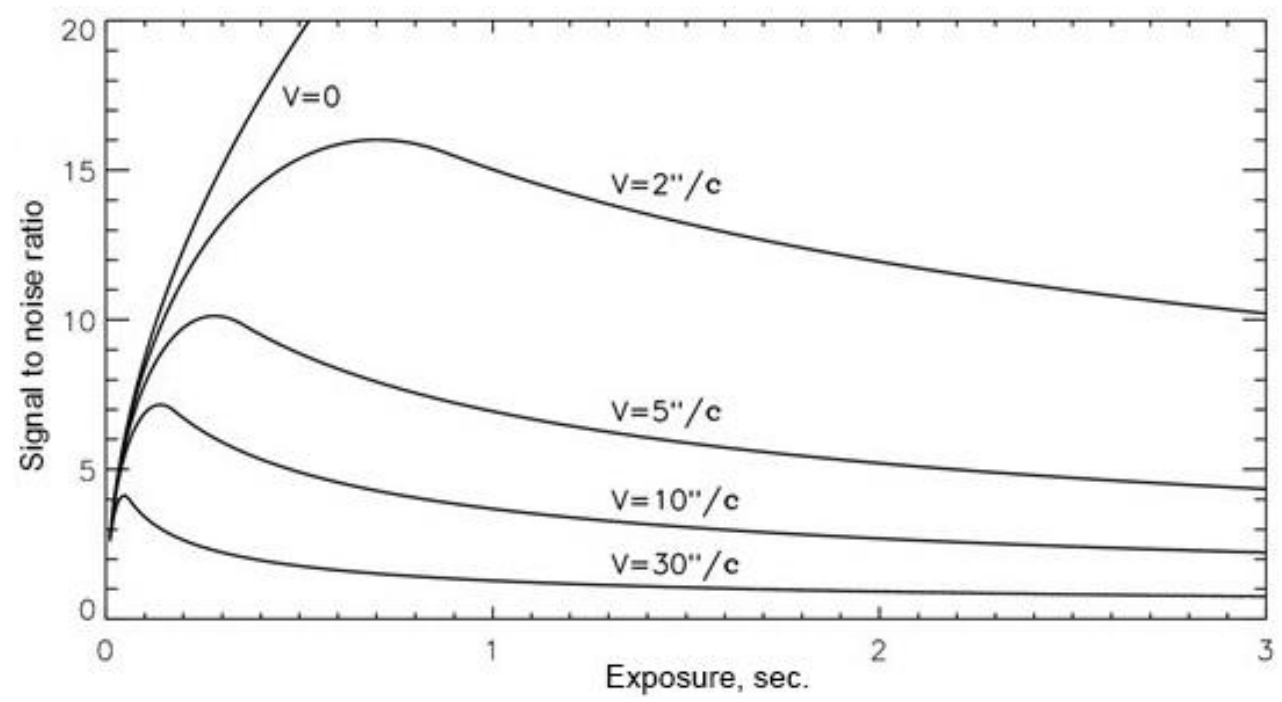

Figure 6. Signal-to-noise ratio as a function of exposure time at different velocities of SO of the 18th magnitude in the field of AZT-33VM

It is seen that at an apparent velocity of $30 " / \mathrm{s}$ for semidiurnal $\mathrm{SO}$ of the 18th magnitude in the field of AZT-33VM the maximum signal-to-noise ratio is close to threshold value 3 , an increase in the exposure time leading to a decrease in the signal-to-noise ratio.

\section{RESULTS OF OBSERVATIONS OF SPACE DEBRIS DETECTED IN THE VICINITY OF GNSS ORBITS}

In 2018, AZT-33VM made observations to detect and catalog the previously unknown small-sized SD objects. The mathematical software available at the telescope allows us to evaluate the situation in the field of the telescope and timely detect objects with unknown orbital parameters. The method of identifying such objects is based on the near-real-time acquisition of preliminary data on apparent angular velocity and position of an observed object, necessary for identifying it with cataloged SOs as well as for further monitoring and measurement of unknown $\mathrm{SO}$ in different portions of the orbit.

During surveys conducted for 87 nights in 2018, AZT-33VM detected 168 previously unknown objects with brightness from 13.8 to 20.5 magnitude. Figure 7 depicts distribution of brightness of SO with unknown orbital parameters, found by AZT-33VM in 2018.

Most detected and cataloged objects are in geosynchronous orbits because local surveys were carried out in areas of the sky near the terrestrial equator. In this case,

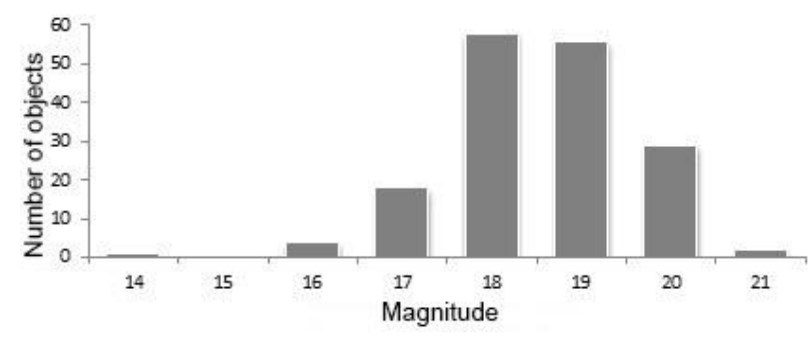

Figure 7. Histogram of brightness distribution of SOs identified with AZT-33VM in 2018 the surveyed region may be crossed by SOs with orbits of other types. One of such objects was discovered in a circular orbit, where GLONASS operates. Duration of the first series of observations was $18 \mathrm{~min}$.

The prediction error the next night was $1.8^{\circ}$ along the orbit, $0.1^{\circ}$ across the orbit. The next nights, the orbital parameters were improved, the object was tracked and cataloged with No. 67403. Parameters of its osculating orbit for 15:22:25 UTC on November 13, 2018 in the coordinate system of the epoch of the date:

- $\quad$ semimajor axis $=25478.9 \mathrm{~km}$,

- eccentricity $=0.0061$,

- inclination $=64.14^{\circ}$,

- longitude of ascending node $=166.69^{\circ}$,

- perigee argument $=139.72^{\circ}$,

- mean anomaly $=331.86^{\circ}$,

- $A M R=0.4 \mathrm{~m}^{2} / \mathrm{kg}$.

Observations with telescopes having high limiting magnitude allow us to investigate characteristics of smallsized SD, in particular they provide detailed light curves for objects of the 17-18th magnitude. The main types of photometric observations are brightnesses as a function of time and phase angle. Light curves of object No. 67403 obtained with AZT-33IK show considerable multipeak variations with amplitude up to 3.5 magnitude, with a pronounced periodicity $T=465.5 \mathrm{~s}$. A light curve on one of the observation dates is exemplified in Figure 8.

Figure 9 shows a folded light curve for a rotation period defined by the Lafler - Kinman method of superimposed epochs [Lafler, Kinman, 1965].

The object was measured at different phase angles (angle in the Sun - space object - observer system). This yielded the phase dependence of brightness in a wide range of phase angles (Figure 10).

Brightness as a function of phase angle is an important characteristic that in many cases allows us to evaluate the shape and size of SO by comparing it with the phase functions calculated for the bodies of simple geometric shapes (sphere, cylinder, plane) [McCue et al., 


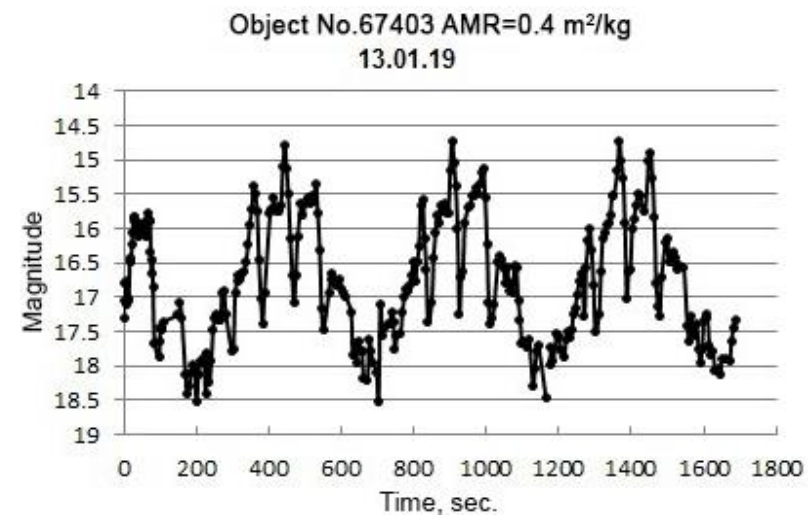

Figure 8. Light curve of an SD object detected with AZT-33VM near GNSS GLONASS orbits

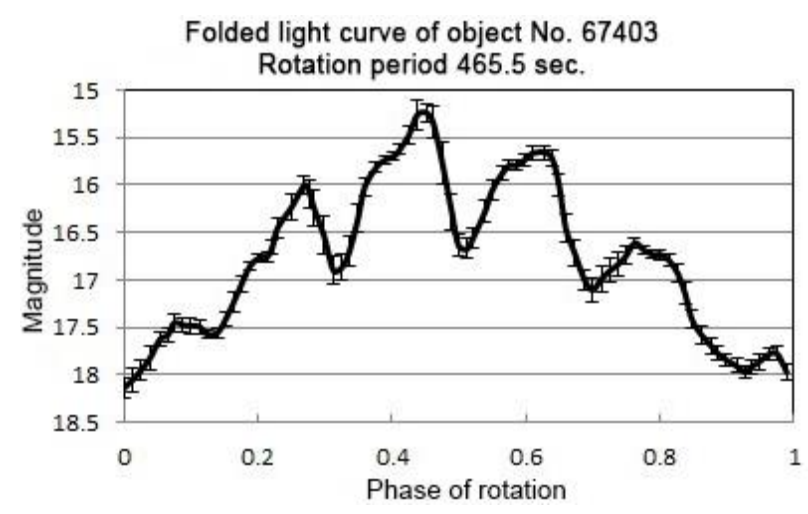

Figure 9. Folded light curve of brightness of object No. 67403 as a function of phase angle of rotation with a period of $465.5 \mathrm{~s}$

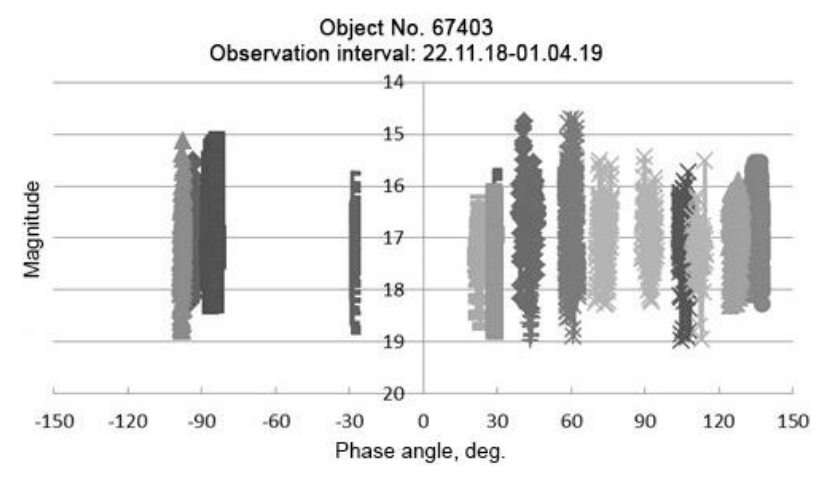

Figure 10. Brightness of object No. 67403 as a function of phase angle

1971]. Lack of phase angle dependence of mean brightness, observed in object No. 67403, in combination with the large amplitude of brightness variations, indicates the predominant shape of specularly reflecting surface (sphere or cylinder) complicated by significant irregularities. Given the mean brightness of an object equal to the 17th magnitude for a specular sphere, from (1) we obtain an estimated object size of $40 \mathrm{~cm}$.

\section{CONCLUSIONS}

Study of the distribution of small-sized SD in nearEarth space is of great importance for safe operation of spacecraft. We have described the new 1.6-m wide- angle telescope AZT-33VM of the ISTP SB RAS Sayan Solar Observatory, which is designed to address fundamental and applied problems of modern astronomy. We have developed a technique for observing and identifying orbital parameters of non-cataloged objects found during optical observations. We have reported the results of SD object detection in the vicinity of GNSS GLONASS. Trajectory parameters and photometric characteristics of the object have been defined.

The work was performed with budgetary funding of Basic Research program II.16. The results were obtained using the equipment of Center for Common Use «Angara» [http://ckp-rf.ru/ckp/3056/].

\section{REFERENCES}

Abalakin V.K., Aksenov E.P., Grebenikov E.A., Demin V.G., Ryabov Yu.A. Spravochnoe rukovodstvo po nebesnoi mekhanike $i$ astrodinamike. Izd. 2 [Reference Guide to Celestial Mechanics and Astrodynamics. 2nd ed.]. Moscow, Nauka Publ., 1976. 273 p. (In Russian).

Ackermann M.R., Kiziah R.R., Beason J.D., Zimmer P.C., McGraw J.T. Exploration of wide-field optical system technologies for sky survey and space surveillance. Proc. 30th Space Symposium. Colorado Springs, Colorado, USA. 2014, pp. 1-28.

Bordovitsyna T.V., Avdyushev V.A. Teoriya dvizheniya iskusstvennykh sputnikov Zemli. Analiticheskie $i$ chislennye metody. Izd. 2. [The Theory of Motion of Artificial Earth Satellites. Analytical and Numerical Methods. 2nd ed.]. Tomsk, TGU Publ. 2016, 254 p. (In Russian).

Chuprakov S.A., Eselevich M.V., Korobtsev I.V. Stray light protection system of the AZT-33VM telescope focal plane, Sayan Astronomical Complex. J. Astronomical Telescopes, Instruments, and Systems. 2018, vol. 4, no. 2, p. 024002. DOI: 10.1117/1.JATIS.4.2.024002.

Denisenko S.A., Kamus S.F., Pimenov Yu.D., Tergoev V.I., Papushev P.G. Fast wide-angle telescope AZT33VM. Opticheskii zhurnal. [J. Optical Technology]. 2009, vol. 79, no. 9, pp. 48-51 (in Russian).

Dick J., Herridge P., Tremayne-Smith R., Davey J., Crowther R. Surveying for debris in MEO with optical sensors. Proc. 5th European Conference on Space Debris, ESA. 2009. vol. 5, iss. 1.

Eselevich M.V., Goryashin V.E., Korobtsev I.V., Tsukker T.G. Observations of unknown space debris objects at AZT33VM telescope. Ekologicheskiy vestnik nauchnykh tsentrov ChES [Ecological Bulletin of Research Centers of the Black Sea Economic Cooperation]. 2017, vol. 4, iss. 3, pp. 52-60. (In Russian). DOI: 10.31429/ vestnik-14-4-3-52-60.

ESA's Annual Space Environment Report. 2019. https:// www.sdo.esoc.esa.int/environment_report/Space_Environment_ Report_latest.pdf (accessed 20 August 2019).

Everhart E. Implicit single-sequence methods for integrating orbits. Celestial Mechanics. 1974, vol. 10, iss. 1, pp. 3555. DOI: 10.1007/BF01261877.

Hoots F.R., Roehrich R.L. Models for propagation of NORAD element sets. Spacetrack Report No. 3. Aerospace Defense Center, Peterson AFB, CO. 1988, 90 p.

Howell S.B. Handbook of CCD Astronomy. Second ed. Cambridge. UK: Cambridge University Press, 2006. 223 p.

Kamus S.F., Tergoev V.I., Papushev P.G., Drujinin S.A., Karavaev Y.S., Palachev Y.M., Denisenko S.A., Lipin N.A. Wide range astronomical telescope. Opticheskiy zhurnal [J. Optical Technology]. 2002, vol. 69, no. 9, pp. 84-87. (In Russian).

Kelecy T., Jah M., Sydney P., Kervin P. Analysis of PanSTARRS photometric and astrometric data for data associa- 
tion and physical consistency assessment. Proc. 6th European Conference on Space Debris. Darmstadt, Germany, 22-25 April 2013 (ESA SP-723), id.28.

Lafler J., Kinman T.D. An RR Lyrae star survey with the Lick 20-inch astrograph II. The calculation of RR Lyrae periods by electronic computer. Astrophys. J. Suppl. 1965, vol. 11, pp. 216. DOI: 10.1086/190116.

Mulrooney M., Matney M., Hejduk M., Barker E. An investigation of global albedo values. Proc. Advanced Maui Optical and Space Surveillance Technologies Conference. 2008, p. E65.

McCue G.A., Williams J.G., Morford J.M. Optical characteristics of artificial satellite. Planetary and Space Sci. 1971, vol. 19, no. 8, pp. 851-868. DOI: 10.1016/00320633(71)90137-1.

Newsletters of PJSC "IAC Vympel”. 2019. http://spacedata. vimpel.ru/ru (accessed 10 January 2019).

Schildknecht T. Optical surveys for space debris. Astron. Astrophys. Rev. 2007, vol. 14, pp. 41-111. DOI: $10.1007 /$ s00159-006-0003-9.

Schildknecht T., Ploner M., Hugentobler U. The search for debris in GEO. Adv. Space Res. 2001, vol. 28, no. 9, pp. 12911299. DOI: 10.1016 / S0273-1177 (01) 00399-4 .

Schildknecht T., Vananti A., Herzog J., Hinze A., Krag H., Flohrer T. Optical surveys for space debris in MEO. Proc. 9th US-Russian Space Surveillance Workshop. 2012. Listvyanka (Irkutsk), Russia.

Seitzer P., Smith R., Africano J., Jorgensen K., Stansbery E., Monet D. MODEST observations of space debris at geosynchronous orbit. Adv. Space Res. 2004, vol. 34, iss. 5, pp. 1139-1142. DOI: 10.1016/j.asr.2003.12.009.

Silha J., Schildknecht T., Hinze A., Flohrer T., Vananti A. An optical survey for space debris on highly eccentric and inclined MEO orbits. Adv. Space Res. 2017, vol. 59, pp. 181192. DOI: 10.1016/j.asr.2016.08.027.

URL: http://ckp-rf.ru/ckp/3056/ (accessed 12 March 2019).

How to cite this article

Korobtsev I.V., Tsukker T.G., Mishina M.N., Goryashin V.E., Eselevich M.V. Observations of space debris in the vicinity of orbits of Global Navigation Satellite Systems. Solar-Terrestrial Physics. 2020. Vol. 6. Iss. 3. P. 94-100. DOI: 10.12737/stp-63202014. 